\title{
Emergence of Additional Visible Range Photoluminescence Due to Aggregation of Cyanine Dye - Astraphloxin on Carbon Nanotubes Dispersed with Anionic Surfactant
}

Petro Lutsyk ${ }^{1,2, *}$, Yuri Piryatinski ${ }^{2}$, Mohammed AlAraimi ${ }^{1,3}$, Raz Arif $^{1,4}$, Mykola Shandura ${ }^{5}$, Oleksiy Kachkovsky ${ }^{6}$, Anatoli Verbitsky ${ }^{2}$, and Aleksey Rozhin ${ }^{1}$

${ }^{1}$ Nanoscience Research Group and Aston Institute of Photonic Technologies, School of Engineering \& Applied Science, Aston University, Aston Triangle, B4 7ET Birmingham, UK;

${ }^{2}$ Institute of Physics, National Academy of Sciences of Ukraine, 46, prospekt Nauky, 03680 Kyiv, Ukraine;

${ }^{3}$ Al Musanna College of Technology, Muladdah, Al Musanna, P.O.Box 191, P.C. 314, Sultanate of Oman.

${ }^{4}$ Physics Department, Faculty of Science, University of Sulaimani, P.O.Box: 334, Sulaimani, IraqKurdistan Region;

${ }^{5}$ Institute of Organic Chemistry, National Academy of Sciences of Ukraine, 5, Murmanska str., 02660 Kyiv, Ukraine;

${ }^{6}$ Faculty of Physics, Taras Shevchenko National University of Kyiv, 64/13, Volodymyrska str., 01601 Kyiv, Ukraine.

* Corresponding author email: p.lutsyk@aston.ac.uk, phone +44 01212045070 


\section{Abstract}

Self-organization of organic molecules with carbon nanomaterials leads to formation of functionalized molecular nano-complexes with advanced features. We present a study of physical and chemical properties of carbon nanotube-surfactant-indocarbocyanine dye (astraphloxin) in water focusing on aggregation of the dye and resonant energy transfer from the dye to the nanotubes. Self-assembly of astraphloxin is evidenced in absorbance and photoluminescence depending dramatically on the concentrations of both the dye and surfactant in the mixtures. We observed an appearance of new photoluminescence peaks in visible range from the dye aggregates. The aggregates characterized with red shifted photoluminescence peaks at 595, 635 and $675 \mathrm{~nm}$ are formed mainly due to the presence of surfactant at the premicellar concentration. The energy transfer from the dye to the nanotubes amplifying near-infrared photoluminescence from the nanotubes is not affected by the aggregation of astraphloxin molecules providing important knowledge for further development of advanced molecular nano-complexes. The aggregation with the turned-on peaks and the energy transfer with amplified photoluminescence create powerful tools of visualization and/or detection of the nanotubes in visible and near-infrared spectral range, respectively, boosting its possible applications in sensors, energy generation/storage, and healthcare. 


\section{Introduction}

One of the most important challenges in nano-science and technology is a construction of photoluminescent (PL) nano-complexes, which can be applied as effective chemical probes, labels for diagnostics and treatment (theranostics) of live tissue, etc. ${ }^{1-7}$ Recently, we have demonstrated a formation of very promising three-component system of single-wall carbon nanotube (SWNT) anionic surfactant - cationic indocarbocyanine dye complexes with enhanced PL properties in nearinfrared (NIR) due to resonant energy transfer (RET) from the dye to the SWNT. ${ }^{8,9}$ Importantly, an electrostatic assembly of the cationic dye and anionic surfactant is essential for the complexation process with RET overcoming the presence of surfactant around SWNT. Besides, this phenomenon has high potential to be used in many other aspects of future development of functionalized nanosystems with tailored properties, like light harvesting devices, energy generation and storage systems, etc. ${ }^{10,11}$

To date, the two-component SWNT-surfactant systems are abundantly studied providing efficient and stable SWNT dispersions in water due to an attraction of hydrophobic parts of the surfactant to the SWNT surface. ${ }^{12-14}$ In the three-component system, a hydrophilic part of the surfactant attracts Coulombically charged part of the ionic dye, e.g. astraphloxin ${ }^{8}$ or dioxaborine terminated polymethine dye. ${ }^{9}$ Nevertheless, it is very simplified picture of the reality requiring deep and comprehensive consideration of $\pi-\pi$ stacking, weak Van der Waals interactions, formation of excimers, exciplexes as well as the dye J- or H-aggregates. ${ }^{15,16}$ Generally, the aggregation results in a considerable coupling of the dipole moments of the aggregated molecules exhibiting in emergence of excitonic bands/transitions in absorbance and PL spectrally shifted from the bands/transitions of the molecular monomers. The narrow red shift bands feature J-aggregates forming 'staircase'-type self-assembly, and blue shift bands are referred to H-aggregates assembled in 'sandwich'-type structures. ${ }^{15,16}$ Besides, the broad red shift bands could be associated with excimers (or exciplexes), short-lived dimers formed of two same (or different) molecules, where at least one molecule is in excited state. The tendency to self-assembly of cyanine dyes ${ }^{15}$ and an effect of various additives 
(surfactants) on the dye aggregation complicates a use of multi-component systems (including our three-component complexes) in real life practice.

Here, the absorption spectroscopy, excitation - emission PL mapping and time-resolved PL analysis allow us to study the complex systems with the purpose to clarify a dramatic influence of the surfactant concentration as well as the dye concentration on the aggregation process in the above complex system of SWNT-surfactant-dye. High concentrations of astraphloxin dye employed in our current investigations provide us strong evidences of J-like-aggregation of astraphloxin (in form of new red shift PL bands). The mechanism of the aggregate formation is discussed concluding the key contribution of the dye and the surfactant interaction.

\section{Experimental Methods and Materials}

Samples under study. The cationic cyanine dye, astraphloxin, was obtained from the Institute of Organic Chemistry (NASU, Ukraine). The concentrations of aqueous solutions of astraphloxin were in the range of $(1-2) \cdot 10^{-5} \mathrm{M}$, where the latter $\left(2 \cdot 10^{-5} \mathrm{M}\right)$ is the dye concentration with strong reabsorption of PL. Consideration of astraphloxin as a model dye in the studies is supported by its unique interaction with SWNTs covered by anionic surfactant, ${ }^{8}$ relatively simple molecular structure, ${ }^{17}$ and low aggregation ${ }^{8,18}$ in solutions of neat dye. Sodium dodecyl-benzene sulfonate (SDBS) (Sigma-Aldrich) was admixed to solutions of astraphloxin at premicellar $(0.05 \mathrm{mg} / \mathrm{ml}$ being below critical micelle concentration (CMC) ) and micellar concentrations $(0.3 \mathrm{mg} / \mathrm{ml}$ - slightly above $\mathrm{CMC}$ ), where, according to the literature, ${ }^{19} \mathrm{CMC}$ for SDBS is approximately $0.15 \mathrm{mg} / \mathrm{ml}$. The choice of SDBS among the various ionic surfactants is justified with high efficiency and prolonged stability in dispersing SWNTs due to the small head group with benzene ring presence, long alkyl chain, ${ }^{13}$ and the high charge in the head group that strengthens the electrostatic repulsion between surface covered SWNTs preventing the aggregation of the tubes. ${ }^{14}$ SDBS attaches to the SWNT mostly due to hydrophobicity of the benzene containing tail of the surfactant. ${ }^{20}$ Unless the bile salts provide highest efficiency in dispersing the SWNT, ${ }^{21} \mathrm{SDBS}$ yields the highest PL amplification in the NIR range for the system of SWNT-surfactant-dye. ${ }^{8}$ 
Purified SWNTs (CoMoCAT, purchased from SWeNT Inc., CG100, lot \#000-0012) were used as a source material to prepare SWNT dispersions. $1.2 \mathrm{mg}$ of SWNTs powder was loaded in $20 \mathrm{ml}$ of deionized water in the presence of $5 \mathrm{mg}$ of anionic surfactant, SDBS. The above mixture was subjected to ultrasonication using a NanoRuptor (Diagenode) processor for $1 \mathrm{~h}$ at $21 \mathrm{kHz}$ and $250 \mathrm{~W}$ to obtain dispersions. The ultrasound treated dispersions were subjected to ultracentrifugation for $2 \mathrm{~h} 30 \mathrm{~min}$ at $17^{\circ} \mathrm{C}$ at $47000 \mathrm{rpm}$ (Beckman Coulter Optima Max-XP, MLS 50 rotor) to remove the aggregate phase and obtain debundled supernatant dispersions of SWNTs. The SWNT of $(6,5),(7,5)$, and $(8,4)$ chiralities are predominant in the obtained dispersions. ${ }^{8}$ To prepare mixtures of SWNTs with astraphloxin, we used 20\% of the initial dispersions of SWNTs. This percentage of the initial SWNT dispersions was chosen to ensure that the PL signal was compatible with the sensitivity limits of the PL detector. The concentrations of specific SWNTs in the mixtures and neat SWNTs for the reference media used in the experiments were the same (20\%). This way, the concentration of SDBS in all samples of the three component mixture (SWNT-SDBS-astraphloxin) was $0.05 \mathrm{mg} / \mathrm{ml}$ (equal to the premicellar concentration of SDBS used in the mixtures). The level of purity for all materials is described in Supporting Information.

Experimental setup. The maps of PL excitation-emission, PLE maps, where the $\mathrm{X}$ axis is the wavelength of PL emission, $\lambda_{\mathrm{EM}}$, and the $\mathrm{Y}$ axis is the wavelength of PL excitation, $\lambda_{\mathrm{EX}}$, are our key experimental data figures. PLE maps with $\lambda_{\mathrm{EX}}$ increment of $5 \mathrm{~nm}$ and PL emission spectra in visible and NIR range at different $\lambda_{\mathrm{EX}}$ in the range of $300-800 \mathrm{~nm}$ were recorded by a Horiba NanoLog excitation-emission spectrofluorometer. The spectrofluorometer was equipped with a silicon detector for visible range and a liquid nitrogen cooled InGaAs array detector for NIR range measurements. 2 and $14 \mathrm{~nm}$ entrance/exit slits were employed for the monochromators in visible and NIR ranges, respectively. For PL excitation in the middle UV range $\left(\lambda_{\mathrm{EX}}=270 \mathrm{~nm}\right)$ the PL emission spectra were measured with USB2000+UV-VIS-ES spectrometer with $600 \mu \mathrm{m}$ optical fiber and with excitation of LLS-270 light-emitting diode (all Ocean Optics B.V.).

The time resolved PL decays were measured with LifeSpec II spectrofluorometer (Edinburgh Instruments Ltd, UK) employing Time Correlated Single Photon Counting (TCSPC) 
with excitation by a picosecond pulse diode laser at $405 \mathrm{~nm}$ (EPL-405). The lifetime data was obtained from exponential fitting via F900 for Windows ${ }^{\circledR}$ software package. The confocal PL images were taken employing the $514 \mathrm{~nm}$ diode laser for the excitation with a confocal microscope system of SP5 TCS II MP made by Leica Microsystems. The absorption spectra were measured with a Lambda 1050 UV/VIS/NIR spectrometer (Perkin Elmer).

\section{Results and Discussion}

We have studied spectral properties of astraphloxin molecules at various concentrations and the effect of SDBS admixture at premicellar and micellar concentrations. Absorption spectra of aqueous solutions of astraphloxin have a maximum at $540 \mathrm{~nm}$ (curve 1 in Figure 1) that characterizes monomers of astraphloxin molecules in water. Small peak at $509 \mathrm{~nm}$ is vibrational progression band of the $540 \mathrm{~nm}$ electronic transition. In addition, the maximum and shape of the astraphloxin absorption spectra do not change in the range of $(0.025-2.5) \cdot 10^{-5} \mathrm{M}^{8}$ thus confirming no aggregation of neat astraphloxin molecules in the above range of concentrations. This corroborates with the previous findings on aqueous solutions of neat astraphloxin starting aggregation at the concentrations above $10^{-3} \mathrm{M}^{18}$

However, according to our experiments, the aqueous solutions of astraphloxin change color and spectral properties (see Supporting Information, Figure S1) at admixture of SDBS depending on the premicellar or micellar concentration of the surfactant added. The absorption spectra for the mixtures of astraphloxin with premicellar concentrations of SDBS have a maximum in the range of dye monomer absorbance $(540 \mathrm{~nm})$ with a moderate additional (non-monomer/aggregate) absorption in the long wavelength (560-600 nm) range (curve 2 in Figure 1). At micellar concentration of SDBS mixed with astraphloxin, we observe absorption maximum at $550 \mathrm{~nm}$ (curve 3 in Figure 1) being shifted by $10 \mathrm{~nm}$ to longer wavelengths relative to the absorption maximum of neat astraphloxin in water. Similar results were observed for various surfactant-dye systems. ${ }^{22,23}$ At the micellar concentration, we consider that micelles form around astraphloxin monomer molecules changing local dielectric permittivity (dielectric screening) of the dye molecules and preventing 
further aggregation. For example, the absorption maximum at $550 \mathrm{~nm}$ is obtained for chloroform solution of astraphloxin having much lower dielectric permittivity $(\varepsilon \approx 4.8)$ comparing to water.
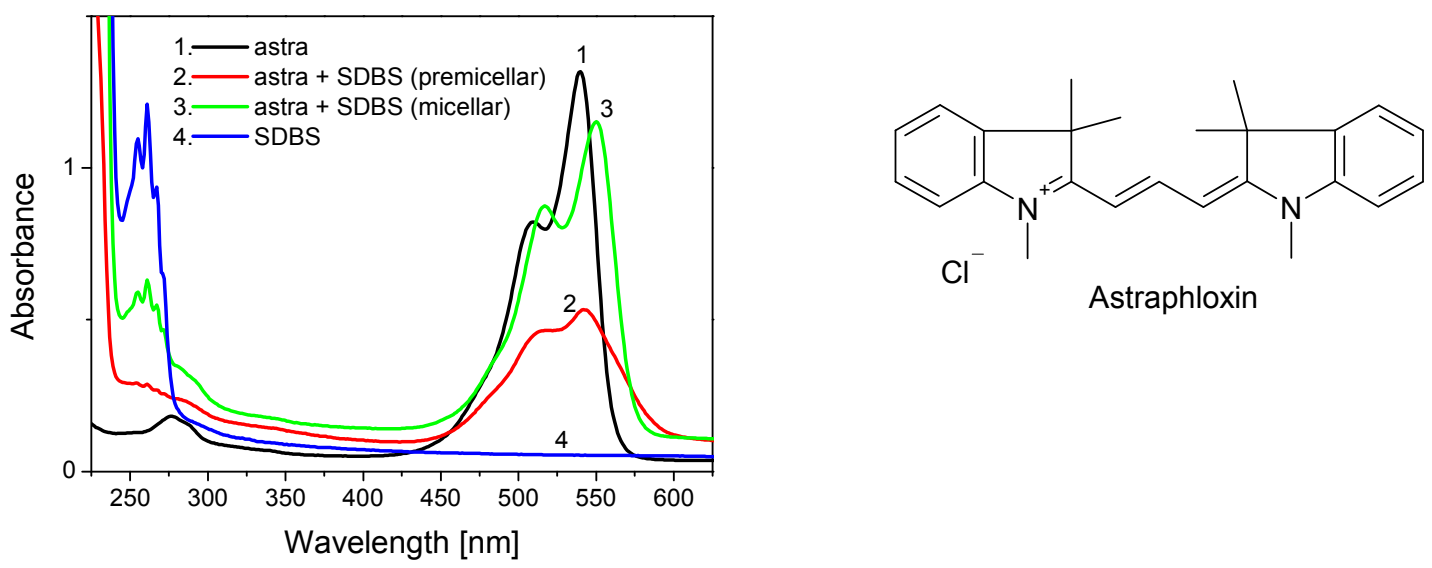

Figure 1. Absorption spectra for aqueous solutions of neat astraphloxin (1), mixtures of astraphloxin with SDBS at premicellar (2) and micellar (3) concentrations, and neat SDBS (4). The concentration of astraphloxin is $1 \cdot 10^{-5} \mathrm{M}$.

Generally, the aggregation of the dye molecules strictly depends on the dye molecular structure and environment (such as $\mathrm{pH}$, solvent polarity, dye concentration, temperature, etc.). ${ }^{15,16}$ Besides, it was shown that the cationic cyanine dyes can have enhanced aggregation in the presence of anionic surfactants at the concentration below the CMC, whereas, at the concentration above the $\mathrm{CMC}$ the anionic surfactant prevents the aggregation of the dye molecules. ${ }^{24}$ Thus, our results on absorption spectra well correlate with the previous findings. ${ }^{15,16,24}$

To understand the nature of the additional absorbance, the PL spectra and PLE maps (Figures 2-5) were studied for various mixtures of astraphloxin with premicellar concentration of SDBS. The PL spectra for the mixtures change in comparison with the PL of neat dye depending on astraphloxin concentration in aqueous solutions. PL spectra of neat astraphloxin have the maximum at $556 \mathrm{~nm}$ (at the dye concentrations up to $1 \cdot 10^{-5} \mathrm{M}$ ), which characterizes astraphloxin monomers in the solution (Figure 2). In the mixture (of astraphloxin at concentration of $1 \cdot 10^{-5} \mathrm{M}$ or lower), PL intensity is slightly (approximately 40\%) quenched in the comparison with neat solution of 
astraphloxin (Figure 2a). Besides, the low intensity PL peak appears at $\lambda_{\mathrm{EX}}=590 \mathrm{~nm}$ and $\lambda_{\mathrm{EM}}=635$ $\mathrm{nm}$ as well as the weak PL shoulders form in the range of $\lambda_{\mathrm{EX}}=450-560 \mathrm{~nm}$ and $\lambda_{\mathrm{EM}}=590-640 \mathrm{~nm}$ (Figure 3a). However, these low PL signals could be observed only for the very fresh mixtures, so the overnight solutions practically do not have above new features resembling neat astraphloxin PLE map (see Supporting Information, Figure S2). The PL peak at $\lambda_{\mathrm{EX}}=590 \mathrm{~nm}$ corresponds to the additional absorbance shoulder in the range of 560-600 nm (Figure 1) and supports a formation of Jlike-aggregates of astraphloxin through interaction of SDBS and astraphloxin molecules. Moreover, the peak at $\lambda_{\mathrm{EX}}=590 \mathrm{~nm}$ corroborates with the peak of J-aggregates formed in Langmuir-Blodgett films of indocarbocyanine molecules with equivalent $\pi$-electron system. ${ }^{25}$ Thus, the new spectral features for the mixtures of astraphloxin with premicellar concentration of SDBS evidence formation J-like aggregation of astraphloxin molecules assisted by the anionic surfactant.

(a)

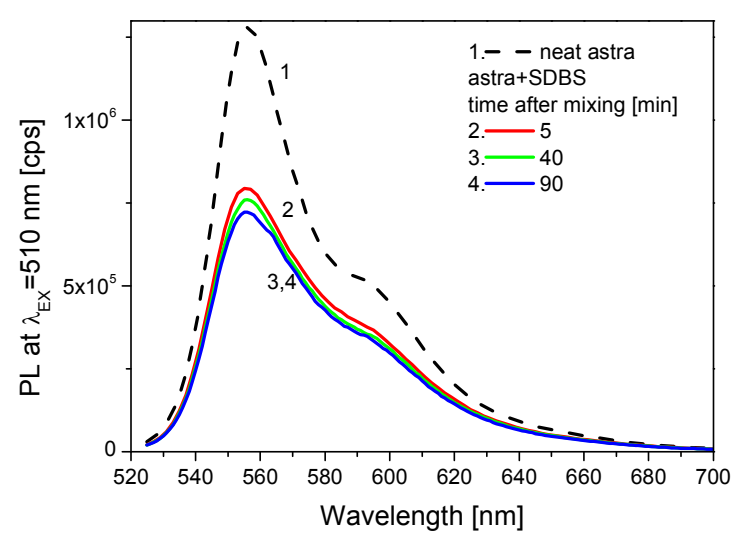

(b)

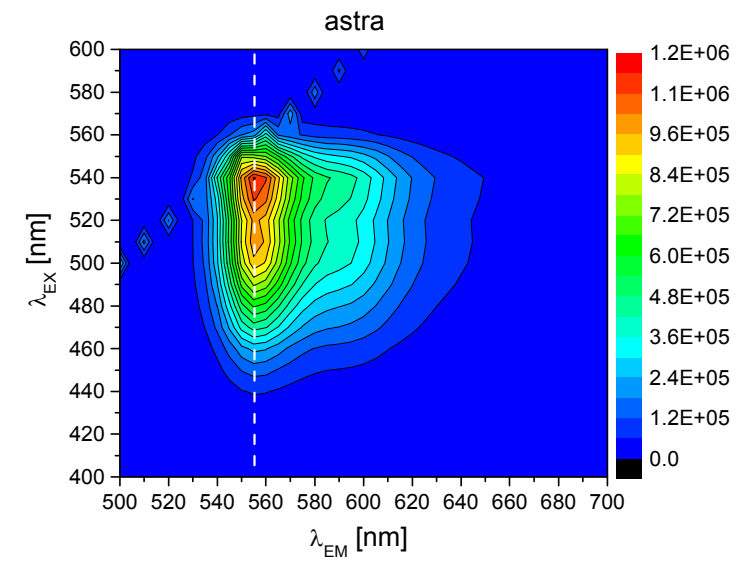

Figure 2. (a) PL spectra at $\lambda_{\mathrm{EX}}=510 \mathrm{~nm}$ for neat astraphloxin (curve 1) and its mixtures with SDBS at premicellar concentration (curves 2-4). Time after the mixing is mentioned on the legend. (b) PLE map for neat astraphloxin, where high PL intensities are decoded in red color, whereas the low intensities are decoded in blue color.

The concentration of astraphloxin is $1 \cdot 10^{-5} \mathrm{M}$. 
The aggregation origin of the new peak $\left(\lambda_{\mathrm{EX}}=590 \mathrm{~nm}\right.$ and $\left.\lambda_{\mathrm{EM}}=635 \mathrm{~nm}\right)$ and weak shoulders $\left(\lambda_{\mathrm{EX}}=450-560 \mathrm{~nm}\right.$ and $\left.\lambda_{\mathrm{EM}}=590-640 \mathrm{~nm}\right)$ depicted in Figure 3a could be clarified due to an increase of the astraphloxin concentration in the mixture up to $2 \cdot 10^{-5} \mathrm{M}$ (Figure $3 \mathrm{~b}$ ). However, the effect of strong reabsorption slightly interferes to measure the correct PL spectra of the dye resulting in the shift of PL peak to longer wavelengths by approximately $3 \mathrm{~nm}$ (to $559 \mathrm{~nm}$ ), but have no effect on long wavelength $(>580 \mathrm{~nm}$ ) parts of the spectral characteristics. As expected, for high concentration of astraphloxin in the mixture, we obtained more prominent aggregation features (PL peaks in the range of $\lambda_{\mathrm{EM}}=595-635 \mathrm{~nm}$ ) and stronger quenching of monomeric emission (PL peak at $\left.\lambda_{\mathrm{EM}}=559 \mathrm{~nm}\right)$. First, new PL peaks at $\lambda_{\mathrm{EM}}=595$ and $635 \mathrm{~nm}$ emerge in the range of excitation of astraphloxin monomer $\left(\lambda_{\mathrm{EX}}=450-560 \mathrm{~nm}\right)$ (Figure $\left.3 \mathrm{~b}\right)$. Second, an intensive peak appears with maximum at $\lambda_{\mathrm{EX}}=590 \mathrm{~nm}$ and $\lambda_{\mathrm{EM}}=635 \mathrm{~nm}$ (Figure $\left.3 \mathrm{~b}\right)$ that was observed a small feature at lower dye concentrations. So, the above aggregation features strongly depend on the dye concentration, being invisible at the astraphloxin concentrations $\leq 5 \cdot 10^{-6} \mathrm{M}^{8}$ become explicit at $2 \cdot 10^{-5} \mathrm{M}$ 
(a)

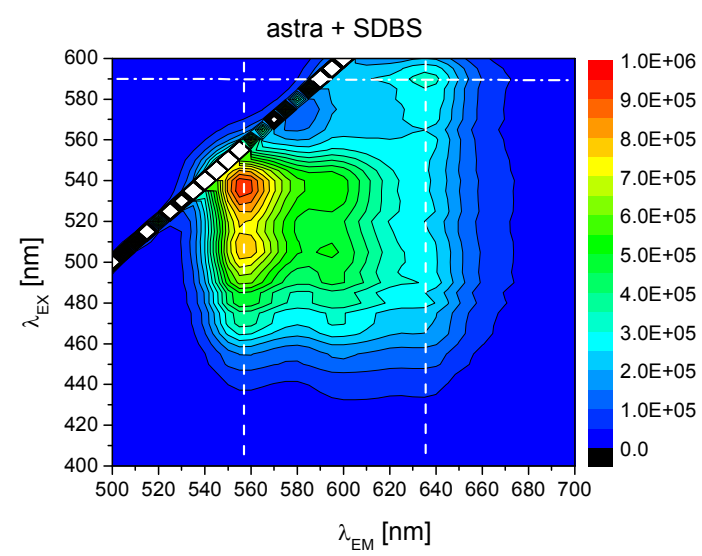

(c)

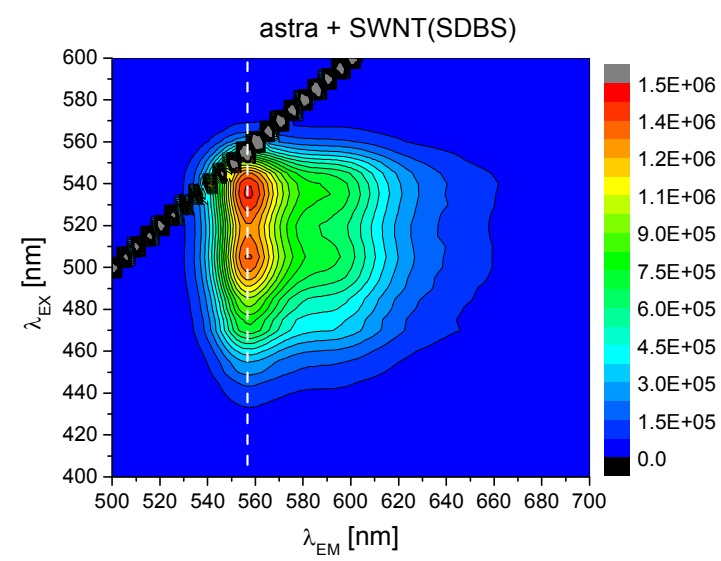

(b)

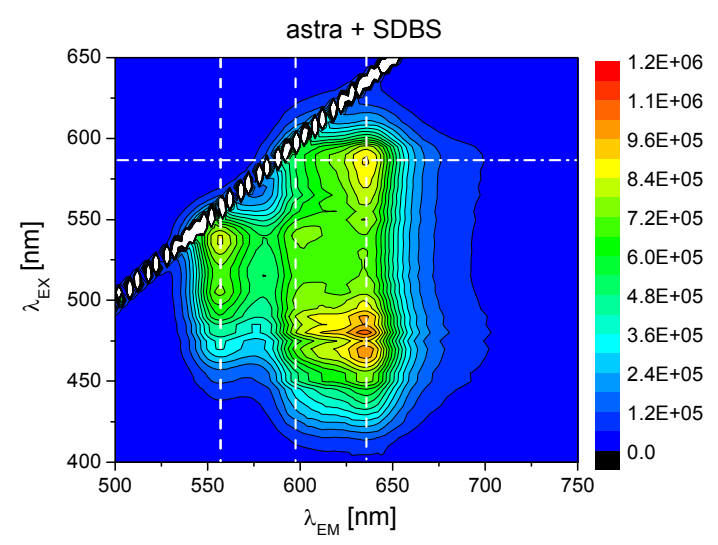

(d)

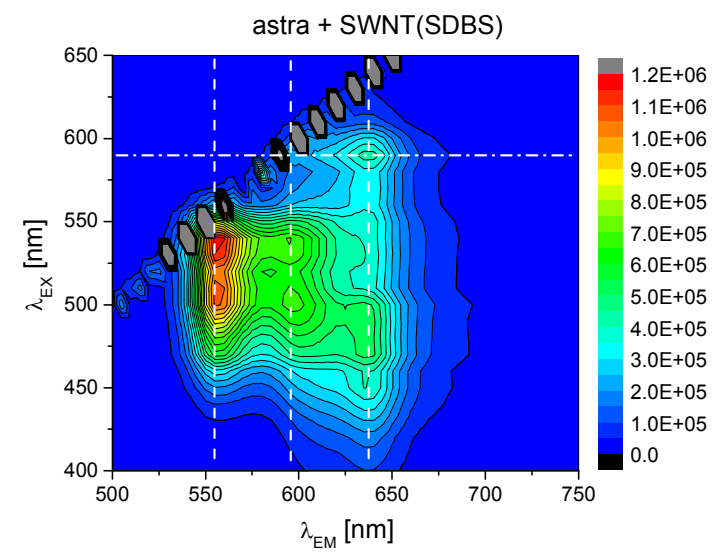

Figure 3. PLE maps for $(a, b)$ fresh mixtures of astraphloxin with SDBS at premicellar concentration and $(\mathrm{c}, \mathrm{d})$ fresh mixtures of astraphloxin with SWNT dispersed via SDBS. The concentration of astraphloxin is $1 \cdot 10^{-5} \mathrm{M}(\mathrm{a}, \mathrm{c})$ and $2 \cdot 10^{-5} \mathrm{M}(\mathrm{b}, \mathrm{d})$. High PL intensities are decoded in red color, whereas low intensities are decoded in blue color.

The aggregation features in PL alter dramatically with time, and, thus, the dynamic behavior of their development was studied (Figure 4). The first aggregate peak at $\lambda_{\mathrm{EM}}=595 \mathrm{~nm}$ appears immediately after the mixing and exceeds the monomer peak $\left(\lambda_{\mathrm{EM}}=559 \mathrm{~nm}\right)$ in a few $(5-10)$ 
minutes (Figure 4a). The second peak at $\lambda_{\mathrm{EM}}=635 \mathrm{~nm}$ grows slowly for the first 15 minutes, and after that, the growing rate accelerates until approximately 80 minutes after the mixing (Figure $4 \mathrm{~b}$ ). In fact, we have seen that the peaks formation is competitive - deceleration of the first peak growth is following by acceleration of the second peak development. In 80 minutes, PL intensity of the peak at $\lambda_{\mathrm{EM}}=635 \mathrm{~nm}$ becomes twice or even one order of magnitude higher, than the peak of the monomer, strongly depending on $\lambda_{\mathrm{EX}}$ (Supporting Information, Figures S3 and S4: $\lambda_{\mathrm{EX}}=400$ $500 \mathrm{~nm}$ and $270 \mathrm{~nm}$, respectively). Within the longer timescales after mixing, the peaks slowly diminish (Figure 4a,b). It should be underlined that the dependence of a ratio of the monomer to the aggregate peaks on $\lambda_{\mathrm{EX}}$ occurs in the range of transparency of astraphloxin monomers. It means a negligibly low absorbance, and as a consequence low PL emission, of the monomers in comparison with the spectral contributions from the aggregates. Thus, at the short wavelength excitation the PL peaks of aggregates become much higher than the monomer bands. 
(a)

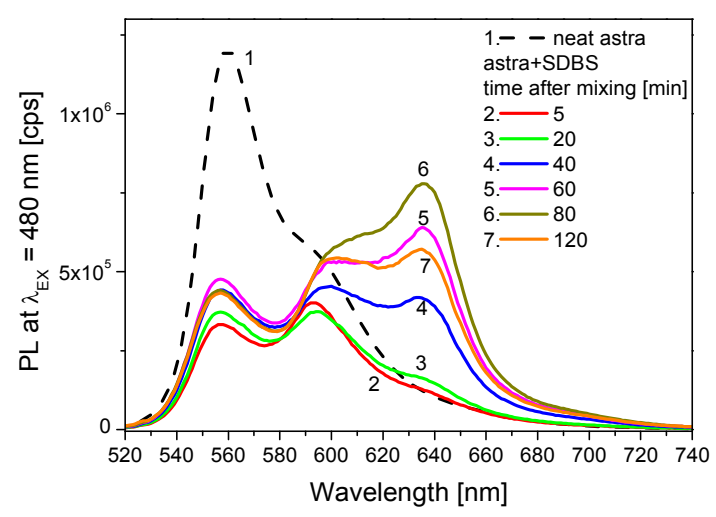

(c)

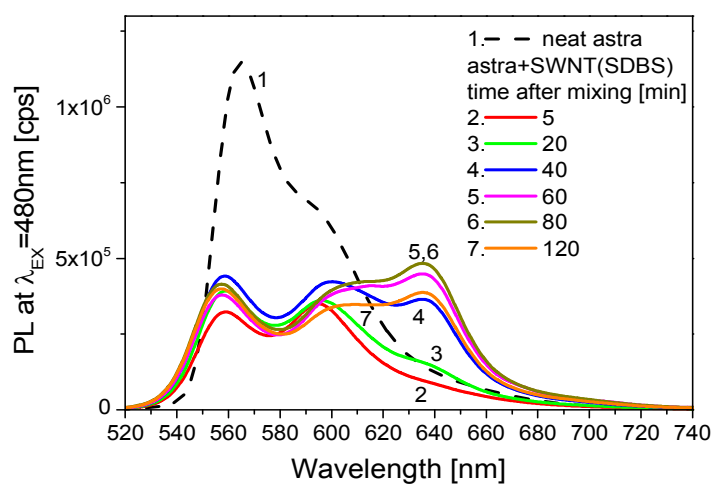

(b)

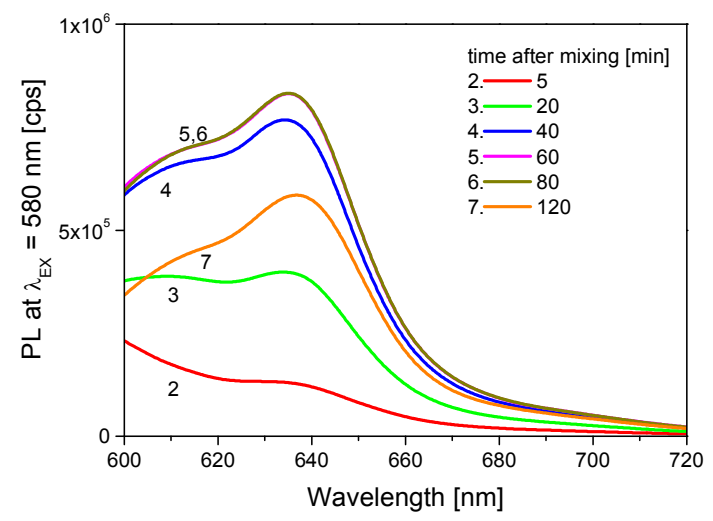

(d)

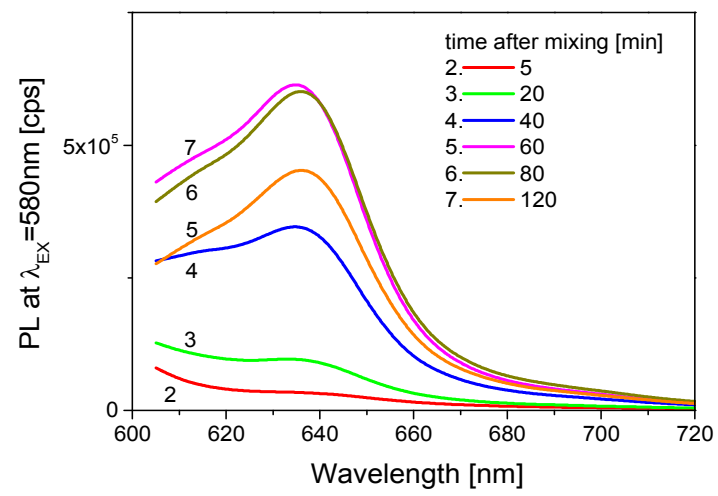

Figure 4. PL spectra at $\lambda_{\mathrm{EX}}=480 \mathrm{~nm}(\mathrm{a}, \mathrm{c})$ and $\lambda_{\mathrm{EX}}=580 \mathrm{~nm}(\mathrm{~b}, \mathrm{~d})$ for neat astraphloxin (curve 1 in a,c), astraphloxin mixtures with SDBS at premicellar concentration (curves 2-7 in a,b) and astraphloxin mixtures with SWNT dispersed via SDBS (curves 2-7 in c,d). Time after the mixing is mentioned on the legends. The concentration of astraphloxin is $2 \cdot 10^{-5} \mathrm{M}$.

So far we have discussed the results of two-component system of dye-surfactant making a comprehensive reference background for the three-component samples of SWNT-surfactant-dye. As the next step, we have studied the mixtures of astraphloxin with SWNT dispersed by SDBS focusing on the aggregation of the dye at the premicellar concentration of SDBS (see Experimental 
section for details). In Figure 3c depicting PLE map, we have no evidence of the dye's aggregation $\left(\lambda_{\mathrm{EX}}=590 \mathrm{~nm}\right)$ in the system with low concentrations of the dye $\left(1 \cdot 10^{-5} \mathrm{M}\right)$ unless identical mixture without SWNT have small signs of the aggregation (Figure 3a). Elevation of astraphloxin concentration up to $2 \cdot 10^{-5} \mathrm{M}$ results in the emergence of aggregation features on PLE map (Figure $3 \mathrm{~d}$ ), but the peaks are less intensive in comparison with the same system without SWNT (Figure $3 b)$. Besides, the PL intensity of astraphloxin monomeric form is $(\sim 30 \%)$ higher in the mixtures with SWNT dispersed by SDBS (Figure 3cd) than that in the mixtures without SWNT (Figure 3ab). Thus, the presence of SWNT slightly reduces the PL from astraphloxin aggregates induced by SDBS. The reduced PL could be explained by two following mechanisms. The first mechanism is PL quenching by the SWNT itself. The second explanation is reasoned by decreased concentration of SDBS that could be involved in the aggregation process for the mixtures with SWNT covered by SDBS. Generally, the temporal development of the new peaks in the system of SWNT-surfactantdye (Figure 4cd) is the same as for astraphloxin mixtures with SDBS only (Figure 4ab). The developed aggregation peaks with strong PL intensity provide us a powerful tool for SWNT visualization.

In addition to strong peaks of aggregates with PL at $\lambda_{\mathrm{EM}}=595$ and $635 \mathrm{~nm}$ (Figures 3-4), we observed non-permanent short-living PL shoulders (bands) in the range of $\lambda_{\mathrm{EM}}=670-770 \mathrm{~nm}$ (Figure 5) at higher concentrations of the dye in the mixtures. In the wide range of $\lambda_{\mathrm{EX}}$ in the PLE maps, the intensity of the bands is extremely small being below of limit for signal registration. However, the explicit peaks at $\lambda_{\mathrm{EM}}=675$ and $750 \mathrm{~nm}$ were registered at short wavelength excitation $\left(\lambda_{\mathrm{EX}}=270 \mathrm{~nm}\right)$ in fresh mixtures (Figure 5), but the peaks start to diminish in several hours after the mixing. At short wavelength excitation, the peaks can be discerned properly due to low contribution of the dye monomer PL in the spectra, like it was shown above for PL peak at $\lambda_{\mathrm{EM}}=595$ and 635 nm (Figures S3 and S4). The low intensity long-wavelength PL features $\left(\lambda_{\mathrm{EM}}=675\right.$ and $\left.750 \mathrm{~nm}\right)$ might characterize a small contribution of unstable aggregates and/or exciplexes/excimers formed in the mixtures. 


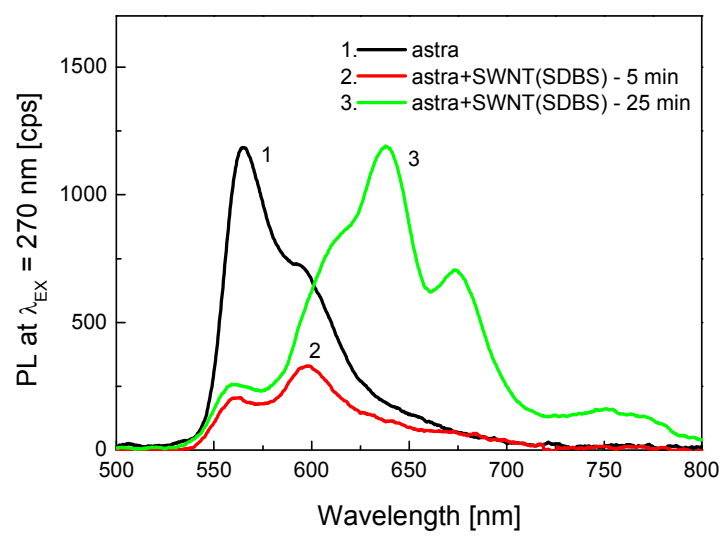

Figure 5. PL spectra at $\lambda_{\mathrm{EX}}=270 \mathrm{~nm}$ for neat astraphloxin (1) and mixtures $(2,3)$ of astraphloxin with SWNT dispersed via SDBS. The concentration of astraphloxin is $2 \cdot 10^{-5} \mathrm{M}$. The time after the mixing for curves 2 and 3 is mentioned on the legends.

All the mixtures of astraphloxin kept overnight have a precipitation at the bottom of the cell and shiny microcrystals floating in the solution (noticeable by naked eye - Figure 6a). Obviously, the higher concentration of astraphloxin is in the mixture, the larger number of microcrystals are formed. The snowflake shaped microcrystals (Figure $6 \mathrm{~b}$ ) have tiny bended elements. It might have some resemblance with curved J-aggregate tubular fibers formed by other carbocyanine dyes ${ }^{26,27}$ being characterized by the very narrow 'J-band'. So it could be suggested that the observed snowflake microcrystals grow involving J-aggregation process. The PL spectra of the microcrystals consist of intensive broad band in the range of 600-650 $\mathrm{nm}$ and small shoulder in the range of 670710nm (Figure 6a). Obtained microcrystals have some spectral resemblance with rodlike microcrystals of pseudoisocyanine consisting of two bands with maxima at 595 and $640 \mathrm{~nm}^{26}$ 
(a)

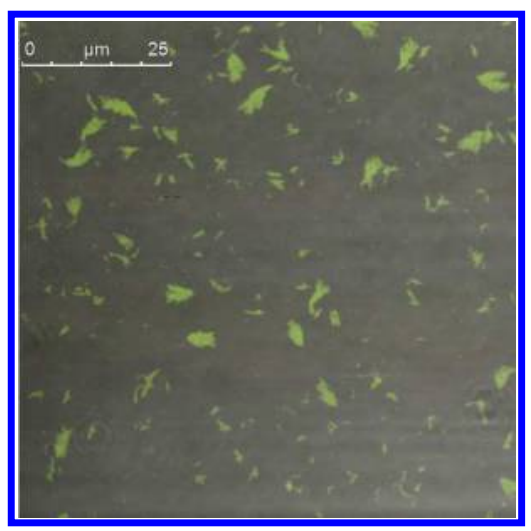

Figure 6. (a) PL spectrum at $\lambda_{\mathrm{EX}}=405 \mathrm{~nm}$ for precipitate microcrystals in the mixture of astraphloxin $\left(2 \times 10^{-5} \mathrm{M}\right)$ with SWNT dispersed via SDBS. For the excitation of microcrystals we used diode laser (EPL-405 in the LifeSpec II system) enabling us to focus precisely on the area of microcrystals precipitation. (inset) Photo of the overnight (in ca. 10 hours after mixing) precipitates in the mixture of SWNT-SDBS-astraphloxin.

The concentration of astraphloxin is $1 \cdot 10^{-5} \mathrm{M}$ (left cuvette) and $2 \cdot 10^{-5} \mathrm{M}$ (right cuvette).

(b) Confocal PL image of precipitate microcrystals in the mixture of astraphloxin $\left(2 \times 10^{-5} \mathrm{M}\right)$ with SWNT dispersed via SDBS.

To sum up the visible range spectral studies on aggregation, we have observed formation of a few types of apparent aggregates of astraphloxin molecules. The first one at $\lambda_{\mathrm{EM}}=595 \mathrm{~nm}$ featuring small red shift relative to monomer emission forms immediately after mixing. So, it can be assigned to small size J-like-aggregates ${ }^{16,25}$ involving the SDBS molecules (as intensity of SDBS PL emission in the range of $320-370 \mathrm{~nm}$ for the mixtures is quenched comparing to neat SDBS peaks - Supporting Information, Figure S4, curves 1,3). The second type of aggregates at $\lambda_{\mathrm{EM}}=635$ $\mathrm{nm}$ forming during one hour after the mixture has to be slow-growing J-like-aggregates without SDBS molecule (as the intensity of SBDS PL emission for the mixtures and neat SDBS is practically the same - Supporting Information, Figure S4, curves 1,4). Besides, the structure of 
aggregates at $\lambda_{\mathrm{EM}}=635 \mathrm{~nm}$ should be predominant in the microcrystals formation and, most probably, represents 'brickwork type' arrangement of the dye molecules. ${ }^{16}$

In our previous work, we have studied the RET from astraphloxin to SWNT energy levels and PL amplification resulted of the RET limiting the experiment to low concentration of astraphloxin. ${ }^{8}$ Herein, we have tested the mixtures at higher astraphloxin content aiming to see the effect of aggregation on the RET. In Figure 7, we compare the PLE maps of neat SWNT dispersed with SDBS and its mixture with astraphloxin at the concentration $2 \cdot 10^{-5} \mathrm{M}$ (see Supporting Information, Figure S5 for absorption spectra). The PLE map of the studied SWNTs dispersions (Figure $7 \mathrm{a})$ in the range of $\lambda_{\mathrm{EX}}$ at $\mathrm{E}_{22}, \mathrm{E}_{33}$, and $\mathrm{E}_{44}(300-700 \mathrm{~nm})$ and $\lambda_{\mathrm{EM}}$ at $\mathrm{E}_{11}(950-1350 \mathrm{~nm})$ features semiconducting SWNTs with chiralities of $(6,5),(7,5)$ and $(8,4)$. Clear evidence of the RET having maximum at $\lambda_{\mathrm{EX}}=560 \mathrm{~nm}$ (Figure $7 \mathrm{~b}$ ) does not coincide with the absorption maxima of the dye monomers $\left(\lambda_{\mathrm{EX}}=540 \mathrm{~nm}\right)$ and the aggregates $\left(\lambda_{\mathrm{EX}}=590 \mathrm{~nm}\right)$. Besides, the higher concentration of astraphloxin gives small red shift of RET $\lambda_{\mathrm{EX}}$ in comparison to low concentrations dye data with $\lambda_{\mathrm{EX}}=555 \mathrm{~nm} .{ }^{8}$ In addition, the PL peaks of the neat SWNT are not quenched in the presence of astraphloxin (see, for example, the PL intensity for $(7,5)$ chirality at $\lambda_{\mathrm{EX}}=650 \mathrm{~nm}$ and $\lambda_{\mathrm{EM}}=1030 \mathrm{~nm}$ in Figure 7), so the dye molecules do not change the intrinsic properties of the SWNT. New data support our former conclusions about the RET from the SWNT-surfactant-dye complex via dipole-dipole interactions between the molecules of astraphloxin and SWNT, where the surfactant remains between the SWNT and the dye. Particularly, the SWNT-SDBS-dye complex can have a substantial overlap of the wave functions of all three components, this way involving Dexter type RET mechanism.

The RET depends on the surfactant packing arrangement on the SWNT surface. According to the literature, ${ }^{20}$ SDBS molecules at the concentrations below $0.1 \mathrm{mM}$ are positioned only in parallel to the SWNT surface, whereas at the concentrations above $0.1 \mathrm{mM}$ the perpendicular orientation of SDBS molecules becomes favorable. The parallel orientation of SDBS allows dye molecule to approach minimum distance to the SWNT surface and to yield efficient RET, whereas the RET at the perpendicular orientation of SDBS molecules is extremely small (according to the 
Förster mechanism, ${ }^{1}$ the RET is limited up to the distance of $9 \mathrm{~nm}$ ). In the studied SWNT dispersions, the SDBS concentration $(0.14 \mathrm{mM})$ is slightly above the $0.1 \mathrm{mM}$. Thus patches of the perpendicularly oriented SBDS start to form on the SWNT surface with prevailed parallel orientation of the SDBS resulting in effective RET from astraphloxin to the SWNT.

(a)

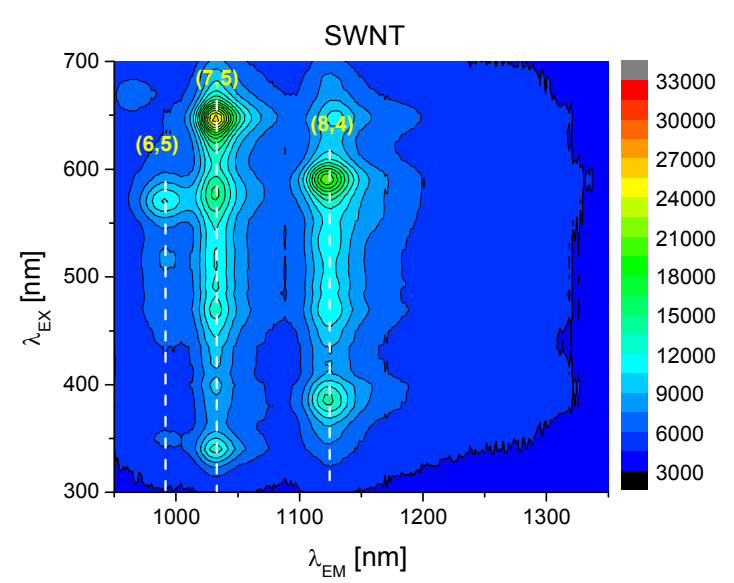

(b)

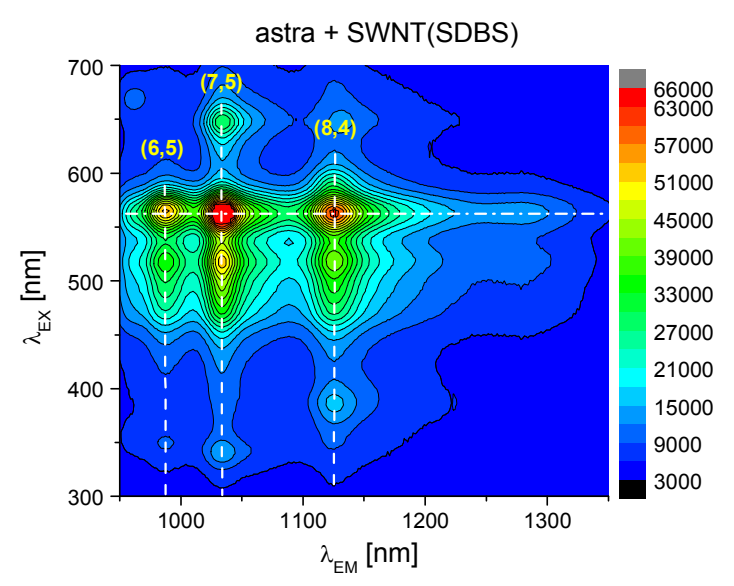

Figure 7. PLE maps of (a) neat SWNT and (b) mixture of astraphloxin $\left(2 \cdot 10^{-5} \mathrm{M}\right)$ with SWNT dispersed via SDBS. High PL intensities are decoded in red color whereas low intensities are decoded in blue color.

Due to the RET at $\lambda_{\mathrm{EX}}=560 \mathrm{~nm}$ (Figure $7 \mathrm{~b}$ ), the maximum PL amplification on the SWNT $(7,5)$ chirality with the factor of approximately 6 is achieved for the dye concentration of $2 \cdot 10^{-5} \mathrm{M}$. In fact, it conforms the data about tendency to saturate the NIR PL from the SWNT and the RET process with an increase of the astraphloxin concentration (Supporting Information, Figure S6). The saturation has been reasoned with limited number of dye molecules able to attach to SWNT.

According to the present study we could also assume that the limitation is rooted in competitive process of the dye aggregation that does not take part in the RET. This assumption relies on the fact that no sign of $590 \mathrm{~nm}$ in $\lambda_{\mathrm{EX}}$ is present in the PLE maps (Figure 7b). Thus, the astraphloxin monomers and aggregates in the mixture do not transfer energy to the nanotubes, whereas only 
astraphloxin complexed to the SBDS covering SWNTs contributes to the RET and NIR visualization of SWNT.

In the reference samples of the astraphloxin-SDBS mixtures with micellar concentration of SDBS (Supporting Information, Figure S7 as an example), the PL bands of astraphloxin monomer have a maximum at $576 \mathrm{~nm}$ (regardless of the dye concentration). This maximum is $20 \mathrm{~nm}$ red shifted in comparison with the monomer peak $(556 \mathrm{~nm})$ of the neat solution of astraphloxin (Figure 2a, curve 1) due to the dielectric screening by SDBS micelles. No aggregate features observed at the premicellar concentration appear in the micellar concentration mixtures, and thus, in overall, this phenomenon correlates with results for other cyanine dyes. ${ }^{24}$ It evidences a formation of micelles around astraphloxin leading to an isolation of the dye molecules and a prevention of possible aggregation. However, the micelle formation does not interfere with the RET from astraphloxin to SWNT with $\lambda_{\mathrm{EX}}=555-560 \mathrm{~nm}$, and hence there are no new features at $\lambda_{\mathrm{EX}}=576 \mathrm{~nm}$ in the PLE maps.

The decays of time-resolved PL (Figure 8) let us to determine lifetimes (an average amount of time the molecular system spent in excited state) of neat astraphloxin, the mixtures of astraphloxin with SDBS and SWNT at various $\lambda_{\mathrm{EM}}$. The lifetimes determined at $\lambda_{\mathrm{EM}}=570 \mathrm{~nm}$ correspond to PL emission of astraphloxin monomers, whereas ones at the long wavelength $\lambda_{\mathrm{EM}}=$ 600,640 and $680 \mathrm{~nm}$ characterize the aggregates. In Table 1, we summarize the lifetime data obtained from the exponential fitting. 
(a)

(b)
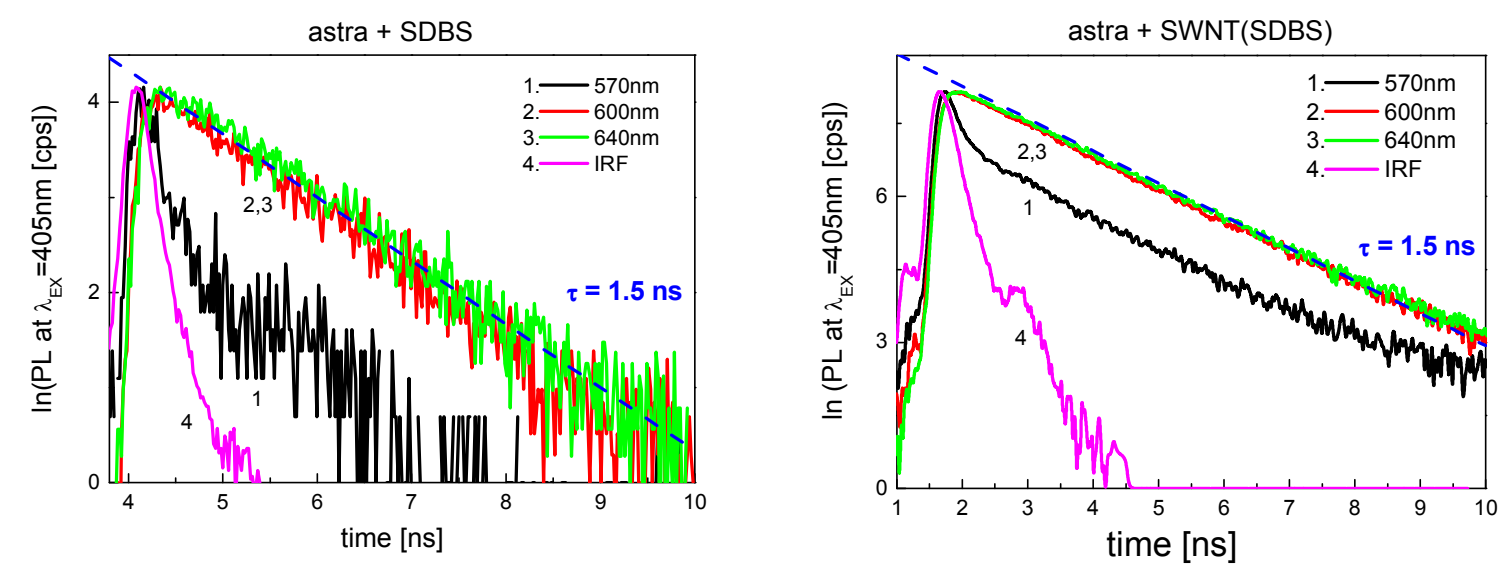

Figure 8. Time-resolved PL decays for mixtures of astraphloxin with SDBS at premicellar concentration (a) and astraphloxin with SWNT dispersed via SDBS at $\lambda_{\mathrm{EM}}=570 \mathrm{~nm}(1), 600 \mathrm{~nm}$ (2), 640 nm (3), and Instrument Response Function (IRF) - (4) obtained with TCSPC technique. The concentration of astraphloxin is $1 \cdot 10^{-5} \mathrm{M}$.

Table 1

Lifetimes of PL for astraphloxin, mixtures of astraphloxin with SDBS, mixtures of astraphloxin with SWNT dispersed via SDBS obtained from exponential fitting of TCSPC PL decays taking into account Instrument Response Function (IRF).

\begin{tabular}{lll}
\hline & $\lambda_{\mathrm{EM}}=570 \mathrm{~nm}$ & $\lambda_{\mathrm{EM}}=600-680 \mathrm{~nm}$ \\
\hline astraphloxin & $\tau=40 \mathrm{ps}$ & - \\
$(300 \mathrm{~K})$ & & \\
\hline astraphloxin & $\tau_{1}=480 \mathrm{ps}(30 \%)$ & - \\
$(77 \mathrm{~K})$ & $\tau_{2}=1.58 \mathrm{~ns}(70 \%)$ & - \\
\hline astraphloxin + SDBS & $\tau=140-250 \mathrm{ps}$ & \\
(micellar concentration) & & $\tau=1.5 \mathrm{~ns}$ \\
\hline astraphloxin + SDBS & $\tau_{1}=40 \mathrm{ps}(60 \%)$ &
\end{tabular}


(premicellar concentration) $\quad \tau_{2}=1.5 \mathrm{~ns}(40 \%)$

$\begin{array}{ll}\text { astraphloxin } \quad \tau_{1}=40 \mathrm{ps}(40 \%) & \tau=1.5 \mathrm{~ns}\end{array}$

$+\mathrm{SWNT}(\mathrm{SDBS}) \quad \tau_{2}=1.5 \mathrm{~ns}(60 \%)$

astraphloxin

$\tau_{1}=1.0 \mathrm{~ns}(>90 \%)$

microcrystals

$\tau_{2}=3.5 \mathrm{~ns}$

The neat astraphloxin has very short lifetime of $40 \mathrm{ps}$ at room temperature $(300 \mathrm{~K})$, and the reason of this is ability to undergo trans-cis photoisomerisation. ${ }^{28,29}$ The flexible structure of the dye monomers having a few stable conformers ${ }^{30}$ provides fast conformational changes and short lifetimes. ${ }^{31}$ The interaction of astraphloxin with SDBS changes the lifetimes depending on the surfactant concentration. At the micellar concentration of SDBS in the mixtures, we obtained increased lifetimes up to $140-250 \mathrm{ps}$. So the micelle formed around the astraphloxin molecule obstructs the isomerization process making the lifetime a factor of 3.5-6 slower. However, the premicellar concentration of SDBS in the mixtures, which provides an efficient aggregation of astraphloxin, results in two component lifetimes. The first one is $40 \mathrm{ps}$, which is predominant at the emission of astraphloxin monomers $\left(\lambda_{\mathrm{EM}}=570 \mathrm{~nm}, 60 \%\right.$ contribution). The second lifetime is much slower having the range of $1.5 \mathrm{~ns}\left(\lambda_{\mathrm{EM}}=570 \mathrm{~nm}, 40 \%\right.$ contribution) and characterizes the range of astraphloxin aggregates ( $\lambda_{\mathrm{EM}}=600-680 \mathrm{~nm} ; 1.5 \mathrm{~ns}$ is a single lifetime component). The emergence of the slower (second) lifetime could be connected with making the molecule more stiff due to creation of steric obstacles for the conformational changes as a result of the aggregation. ${ }^{28,29,31}$ The lifetime for the mixture of astraphloxin with SWNT dispersed via SDBS at $\lambda_{\mathrm{EM}}=680-700 \mathrm{~nm}$ is also approximately $1.5 \mathrm{~ns}$ that associates these peaks more to formation of some J-like-aggregates rather than excimers/exciplexes having characteristic lifetimes of dozen nanoseconds. ${ }^{3}$ However, the broad peak with maximum approximately $\lambda_{\mathrm{EM}}=750 \mathrm{~nm}$ has the lifetime of $2.6 \mathrm{~ns}$, which can be associated with formation of the dye excimers or exciplexes of the dye and SDBS. 
The emergence of second slow lifetime is similar to the 'freezing' of the molecule at liquid nitrogen temperature. The lifetime of astraphloxin obtained at low temperature $(77 \mathrm{~K})$ reflects its radiative lifetime, whereas the photoisomerisation is largely obstructed. The PL decay for neat astraphloxin at $77 \mathrm{~K}$ (Supporting Information, Figure S8) is well fitted with two lifetime components at $\tau_{1}=480 \mathrm{ps}(30 \%$ contribution $)$ and $\tau_{2}=1.58 \mathrm{~ns}(70 \%$ contribution $)$. The formation of microcrystals have two distinctive lifetime components of 1.0 and $3.5 \mathrm{~ns}$ with essential predominance of the first (faster) one (Supporting Information, Figure S9).

\section{Conclusions}

The study of interaction mechanisms between astraphloxin, surfactant and SWNT in water substantially strengthens the implementation of the new approach ${ }^{8}$ for detection of SWNT by cyanine dyes. In this paper we described comprehensive physical and chemical study of the threecomponent system of SWNT-SDBS-astraphloxin focusing on the aggregation of the astraphloxin dye. Although we see strong PL of astraphloxin monomers in the systems, new features of astraphloxin self-assembly appear depending dramatically on the concentrations of both astraphloxin and SDBS in the mixtures. To underline the most important results, it should be mentioned that we have evidenced the new peaks of red shifted PL emission featuring formation various types of J-like-aggregates (including excimers and/or exciplexes) of astraphloxin in presence of SWNT dispersed with SBDS as well as only SDBS at premicellar concentration. The Jlike-aggregates characterized with PL emission peaks of 595, 635 and $675 \mathrm{~nm}$ are formed due to mediation of premicellar SDBS. The weak PL band at $750 \mathrm{~nm}$ features formation of the dye excimers or exciplexes of the dye and SDBS. The micellar concentration of SDBS does not result in the astraphloxin aggregation. The aggregation with $635 \mathrm{~nm}$ PL peak seems to be predominant in the formation of astraphloxin microcrystals forming in the overnight mixtures. The aggregates and microcrystals have a characteristic radiative lifetime components in the range of single nanoseconds, which is two orders of magnitude slower than astraphloxin monomer lifetime (40 ps). 
NIR RET in the complexes of SWNT-SDBS-dye is not affected by formation of the aggregates $(590 \mathrm{~nm})$ and the micelles of SDBS preventing the aggregation of astraphloxin (576 $\mathrm{nm})$. Besides, considering the facts that SDBS perform main role in the aggregation, the microcrystals observed are mostly formed independently of three-component complexes of SWNTSDBS-dye with the RET. Here, we provide the knowledge about new phenomena appearing in the three-component system, which could be used for development of more efficient probe for SWNT detection and/or visualization of the SWNT as well as a platform in the design and applications of tailorable molecular structure nano-assemblies.

Supporting Information. The purity of used materials, supplementary absorption spectra (Figure S1, S5), PLE map (Figure S2), PL spectra (Figure S3, S4, S7), dependence of PL amplification due to the RET on the astraphloxin concentration (Figure S6), and time-resolved PL decays (Figure S8, S9).

\section{Acknowledgements}

The work was supported by NATO SPS project (NUKR.SFPP \#984189) and EU FP 'Horizon2020’ Marie Skłodowska-Curie Individual Fellowship (FOC4SIP, \#654733). M.A. acknowledges the support from the Ministry of Higher Education, Sultanate of Oman. We thank Charlotte Bland and Aston Research Centre for Healthy Ageing (ARCHA) for use of the confocal microscope facilities.

\section{References:}

(1) Lackowicz, J. R. Principles of Fluorescence Spectroscopy; $3^{\text {rd }}$ edition, Springer US, 2006.

(2) Gilmore, A. M. Luminescence: The Instrumental Key to the Future of Nanotechnology; CRC Press: Boca Raton, FL, 2013.

(3) Demchemko, A. P. Introduction to Fluorescence Sensing; Springer: Netherlands, 2015. 
(4) Chowdhury, S.; Wu, Z.; Jaquins-Gerstl, A.; Liu, S.; Dembska, A.; Armitage, B. A.; Jin, R.;

Peteanu, L. A. Wavelength Dependence of the Fluorescence Quenching Efficiency of Nearby

Dyes by Gold Nanoclusters and Nanoparticles: The Roles of Spectral Overlap and Particle

Size. J.Phvs. Chem.C 2011, 115, 20105-20112.

(5) Xu, Y.; Li, Zh.; Malkovskiy, A.; Sun, Sh.; Pang. Y. Aggregation Control of Squaraines and Their Use as Near-Infrared Fluorescent Sensors for Protein. J. Phys. Chem. B 2010, 114, 8574-8580.

(6) Movia, D.; Canto, E. D.; Giordani S. Purified and Oxidized Single-Walled Carbon Nanotubes as Robust Near-IR Fluorescent Probes for Molecular Imaging. J. Phys. Chem. C 2010, 114, 18407-18413.

(7) Lee, J.; Kim, J.; Ahmed, S. R.; Zhou, H.; Kim, J.-M.; Lee. J. Plasmon-Induced Photoluminescence Immunoassay for Tuberculosis Monitoring Using Gold-NanoparticleDecorated Graphene. ACS Appl. Mater. Interfaces 2014, 6, 21380-21388.

(8) Lutsyk, P.; Arif, R.; Hruby, J.; Bukivskyi, A.; Vinijchuk, O.; Shandura, M.; Yakubovskyi, V.; Kovtun, Y.; Rance, G. A.; Fay, M., et al. A Sensing Mechanism for the Detection of Carbon Nanotubes Using Selective Photoluminescent Probes Based on Ionic Complexes with Organic Dyes. Light: Sci. Appl. 2016, 4, e16028.

(9) Shandura, M. P.; Kovtun, Yu. P.; Yakubovskyi, V. P.; Piryatinski, Yu. P.; Lutsyk, P. M.; Perminov, R. J.; Arif, R. N.; Verbitsky, A. B.; Rozhin, A. Dioxaborine Dyes as Fluorescent Probes for Amines and Carbon Nanotubes. Sens. Lett. 2014, 12, 1361-1367.

(10) Levitsky, I. A.; Euler, W. B.; Karachevtsev, V. A. Photophysics of Carbon Nanotubes Interfaced with Organic and Inorganic Materials; Springer: London, 2012.

(11) De Volder, M. F. L.; Tawfick, S. H.; Baughman R. H.; Hart, A. J. Carbon Nanotubes: Present and Future Commercial Applications. Science 2013, 339, 535-539.

(12) Wang, H. Dispersing Carbon Nanotubes Using Surfactants. Curr. Opin. Colloid Interface Sci. 2009, 14, 364-371. 
(13) Islam, M. F.; Rojas, E.; Bergey, D. M.; Johnson, A. T.; Yodh, A. G. High Weight Fraction Surfactant Solubilization of Single-Wall Carbon Nanotubes in Water. Nano Lett. 2003, 3, $269-273$.

(14) Tan, Y. Q.; Resasco, D. E. Dispersion of Single-Walled Carbon Nanotubes of Narrow Diameter Distribution. L.Phvs. Chem.B 2005, 109, 14454-14460.

(15) Steiger, R.; Pugin, R.; Heier, J.; J-aggregation of Cyanine Dyes by Self-Assembly. Colloids Surf. B 2009, 74, 484-491.

(16) Behera, G. B.; Behera, P. K.; Mishra, B. K. Cyanine Dyes: Self Aggregation and Behaviour in Surfactants. A Review, J. Surface Sci. Technol. 2007, 23, 1-31.

(17) Bricks, J. L.; Kachkovskii, A. D.; Slominskii, Y. L.; Gerasov, A. O.; Popov, S. V. Molecular Design of Near Infrared Polymethine Dyes: A Review, Dves Pigments 2015, 121, 238-255.

(18) Ecker, H. Das Absorptionsspektrum von reversiblen Polymerisaten aus Chinolinfarbstoffen. Kolloid-Z. 1940, 92, 35-70. (In German)

(19) Weiss, E.; Groenen-Serrano, K.; Savall, A. Electrochemical Mineralization of Sodium Dodecylbenzenesulfonate at Boron Doped Diamond Anodes, J. Appl. Electrochem. 2007, 37, $1337-1344$.

(20) Matarredona, O.; Rhoads, H.; Li, Z.; Harwell, J. H.; Balzano, L.; Resasco, D. E. Dispersion of Single-Walled Carbon Nanotubes in Aqueous Solutions of the Anionic Surfactant NaDDBS, J.Phvs. Chem. B 2003, 107, 13357-13367.

(21) Wenseleers, W.; Vlasov, I. I.; Goovaerts, E.; Obraztsova, E. D.; Lobach, A. S.; Bouwen, A. Efficient Isolation and Solubilization of Pristine Single-Walled Nanotubes in Bile Salt Micelles, Adv.Eunct.Mater. 2004, 14, 1105-1112.

(22) Duman, O.; Tunc, S.; Kanc1 B. Spectrophotometric Studies on the Interactions of C.I. Basic Red 9 and C.I. Acid Blue 25 with Hexadecyltrimethylammonium Bromide in Cationic Surfactant Micelles. Eluid Phase Eauilibr. 2011, 301, 56-61. 
(23) Tunc, S.; Duman, O.; Kanc1 B. Spectrophotometric Investigation of the Interactions Between Cationic Dye (C.I. Basic Yellow 2) and Anionic Surfactant (Sodium Dioctylsulfosuccinate) in the Premicellar and Micellar Region. Dves Pigments 2012, 94, 233-238.

(24) Mchedlov-Petrossyan, N. O.; Shapovalov, S. A.; Koval, V. L.; Shakhverdov, T. A.; Bochkaryov, Yu. A. The Surfactant-Induced Formation of J- and H-Aggregates in Aqueous Pseudoisocyanine Solutions, Dves Pigments 1992, 19, 33-40.

(25) Debnath, P.; Chakraborty, S.; Deb, S.; Nath, J.; Bhattacharjee, D.; Hussain, S. A. Reversible Transition between Excimer and J-Aggregate of Indocarbocyanine Dye in Langmuir-Blodgett (LB) Films. J.Phvs. Chem.C 2015, 119, 9429-9441.

(26) Lebedenko, A. N.; Guralchuk, G. Ya.; Sorokin, A. V.; Yefimova, S. L.; Malyukin, Y. V. Pseudoisocyanine J-Aggregate to Optical Waveguiding Crystallite Transition: Microscopic and Microspectroscopic Exploration. J.Phvs. Chem. B 2006, 110, 17772-17775.

(27) Kirstein, S.; Daehne. S. J-Aggregates of Amphiphilic Cyanine Dyes: Self-Organization of Artificial Light Harvesting Complexes. Int. J. Photoenergy 2006, 2006, 20363.

(28) Chibisov, A. K.; Zakharova, G. V.; Goerner, H.; Sogulyaev, Yu. A.; Mushkalo, I. L.; Tolmachev, A. I. Photorelaxation Processes in Covalently Linked Indocarbocyanine and Thiacarbocyanine Dyes. J.Phvs. Chem. 1995, 99, 886-893.

(29) Aramendia, P. F.; Negri, R. M.; San Roman, E. Temperature Dependence of Fluorescence and Photoisomerization in Symmetric Carbocyanines. Influence of Medium Viscosity and Molecular Structure J.Phvs. Chem. 1994, 98, 3165-3173.

(30) Boom, K.; Müller, M.; Stein, F.; Ernst, St.; Morgenstern K. Adsorption of a Switchable Industrial Dye on $\mathrm{Au}(111)$ and $\mathrm{Ag}(111)$. L.Phvs. Chem.C 2015, 119, 17718-17724.

(31) Sanborn, M. E.; Connolly, B. K.; Gurunathan, K.; Levitus M. Fluorescence Properties and Photophysics of the Sulfoindocyanine Cy3 Linked Covalently to DNA. J. Phys. Chem. B 2007, 111, 11064-11074. 
Table of Contents Image (3.75 x 1.75 in.)

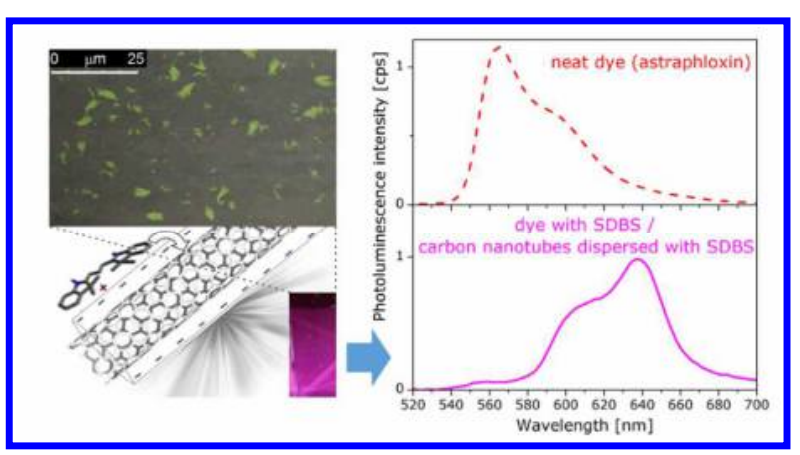

1

2

3

4

5

6

10

12

13

14

15

16

17

18

19

20

21

22

23

24

25

26

27

28

29

30

31

32

33

34

35

36

37

38

39

40

41

42

43

44

45

46

47

48

49

50

51

52

53

54

55

56

57

58

59

60 

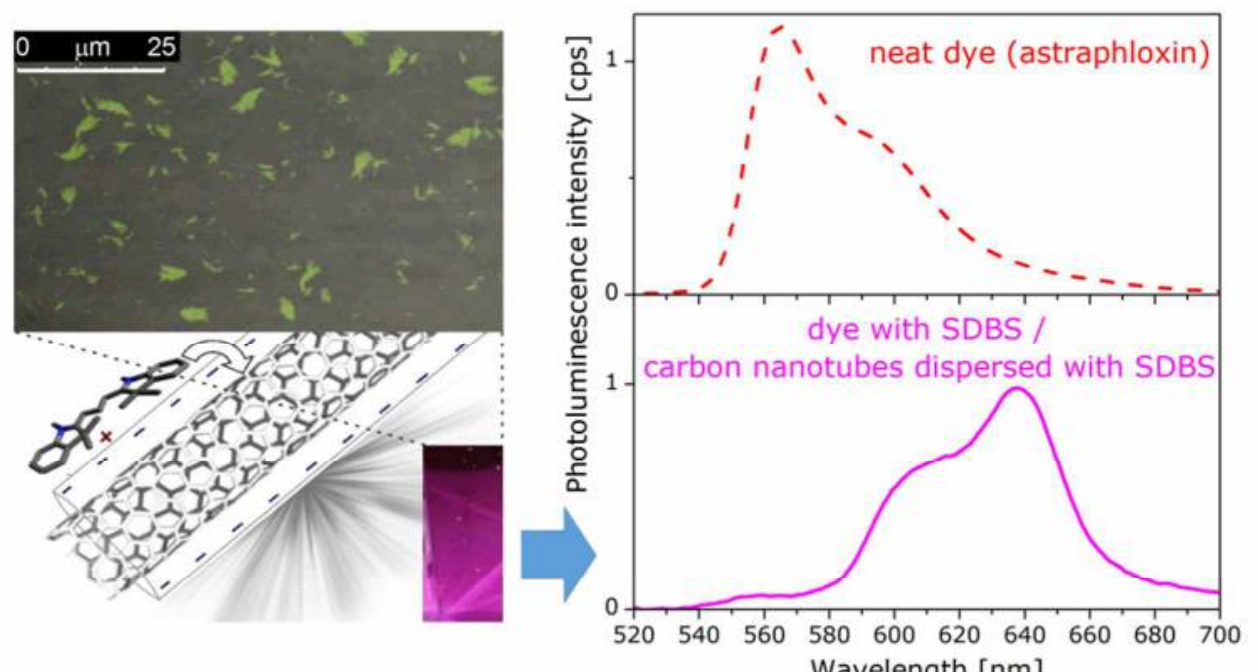

Table of Contents Image

$82 \times 44 \mathrm{~mm}(300 \times 300$ DPI $)$ 